\title{
PRODUÇÃO DE LIPASE EXTRACELULAR DO FUNGO Metarhizium anisopliae
}

\author{
L. M. DUARTE ${ }^{1 *}$, E. H. S. OLIVEIRA ${ }^{1}$, G. R. JESUS ${ }^{1}$, T. L. ABREU-LIMA ${ }^{1}$ e L. M. \\ GRAJALES $^{1 * *}$ \\ ${ }^{1}$ Universidade Federal do Tocantins, Engenharia de Alimentos \\ E-mail para contato: * laiane art@hotmail.com e**grajales@uft.edu.br
}

\begin{abstract}
RESUMO - A presente pesquisa dá suporte ao desenvolvimento de um biorreator de bandejas para a produção do fungo entomopatogênico Metarhirzium anispliae por Fermentação em Estado Sólido, utilizando resíduos agroindustriais como substratos. $\mathrm{O}$ objetivo deste trabalho foi produzir lipases do fungo $M$. anisopliae utilizando bagaço de coco de babaçu e arroz tipo I como suportes à fermentação. Fermentações preliminares foram realizadas para determinar a concentração de suporte a ser utilizado. As fermentações foram realizadas em frascos de erlenmeyer com $10 \mathrm{~g}$ de meio de cultura, a $28^{\circ} \mathrm{C}$ durante 14 dias. Posteriormente, a atividade lipolítica (U. mL-1) foi determinada pelo método titulométrico. De forma geral, as fermentações realizadas revelaram que o tratamento somente com arroz tipo I teve maior atividade lipolítica, com valor de $8,33 \mathrm{U}$. mL-1, e a mistura bagaço do coco babaçu - arroz tipo I, em relação 8:2, teve uma atividade lipolítica considerável de 5,57 U. mL-1.
\end{abstract}

\section{INTRODUÇÃO}

As lipases (triacilglicerol éster hidrolases, EC 3.1.1.3) são enzimas que catalisam, entre outras reações, a hidrólise das ligações éster de lipídeos gerando álcoois e ácidos graxos (Freire et al., 1997). As lipases de origem microbiana constituem um importante grupo de enzimas com alto potencial biotecnológico no setor industrial, porém sua aplicação é bastante reduzida devido ao seu alto custo de produção (Colla et al., 2012). Esta poderia se tornar economicamente viável se realizada por processos de Fermentação em Estado Sólido (FES) (Castilho et al.,2000), no qual o crescimento dos microrganismos se realiza sobre substratos sólidos (resíduos agroindustriais) e sem a presença de água livre, minimizando assim, os custos de produção (Oliveira e Pinotti., 2015). Outra vantagem apresentada por este tipo de processos é a economia de espaço para a realização das fermentações e a simplicidade dos equipamentos utilizados (Mahadik et al., 2002).

A cadeia produtiva do babaçu (Atalleia spp.) é uma das mais representativas do extrativismo vegetal no Brasil, em razão da área de abrangência da palmeira babaçu (13 a 18 milhões de hectares em 279 municípios, situados em 11 Estados), bem como das inúmeras potencialidades e atividades econômicas que podem ser desenvolvidas a partir dela (Carrazza et al.,2012). Os resíduos gerados após o processamento do babaçu podem ser aproveitados como substratos em processos FES, pois a matéria orgânica presente neste material pode ser 
usada como fonte de energia para o crescimento de microrganismos e para a síntese de biomassa celular e dos produtos do metabolismo microbiano (Santana, 2012).

Por outra parte, o fungo filamentoso Metarhizium anisopliae é um bom produtor proteases, quitinases e lipases (Saxena et al., 1999; Silva, 2005). Silva et al (2005) e Shaukat (2009) realizaram estudos utilizando o fungo M. anisopliae, a partir de diferentes fontes de lipídeos e encontraram uma boa produção de lipases. Assim, é possível supor que o binômio microrganismo-substrato: $M$. anisopliae-bagaço de babaçu, possa se tornar um sistema produtor de lipases promissório. Pelos motivos mencionados, este trabalho propõe analisar a produção de lipases utilizando o fungo Metarhizium anisopliae e empregando resíduos de coco de babaçu e arroz tipo I como substratos.

\section{MATERIAIS E MÉTODOS}

\subsection{Materiais}

Microrganismo: O fungo entomopatogênico M. anisopliae cepa ICBC 425 foi adquirido sob a forma de esporos puros em pó e foram armazenados à temperatura de $-4{ }^{\circ} \mathrm{C}$. A cepa foi comprada no laboratório Biocontrol, localizada na cidade de Sertãozinho-SP. A repicagem do microrganismo foi feita por um período de 7 dias a $28^{\circ} \mathrm{C}$ no meio de batata-dextrose-ágar.

Bagaço do coco de babaçu: o bagaço foi o resultado final do processo de compressão da retirada do sumo da amêndoa do coco babaçu e armazenado em freezer a $-12{ }^{\circ} \mathrm{C}$ até o momento da sua utilização.

Arroz tipo I: O arroz Tipo I foi adquirido em beneficiadora de grãos localizada na cidade de Palmas-TO.

\subsection{Metodologia}

Obtenção do inóculo: A cepa foi cultivada em meio de batata-dextrose-ágar (BDA) em erlenmeyers de $250 \mathrm{~mL}$, fechados com tampões de algodão revestidos por tecido do tipo "tecido não tecido" (TNT) e esterilizados a $121^{\circ} \mathrm{C}$ por 20 minutos. Após, o meio solidificar, com os frascos inclinados, o fungo foi incubado durante 07 dias em câmara climatizada BOD marca Adamo à temperatura de $28 \pm 1{ }^{\circ} \mathrm{C}$.

Preparação da solução nutriente e suspensão inoculante: Após 07 dias de incubação, foi preparada a suspensão de conídios adicionando ao erlenmeyer uma solução nutriente, previamente esterilizada, composta por $100 \mathrm{~mL}$ de água destilada, 0,065g de Cloreto de Potássio $(\mathrm{KCl}), 0,033 \mathrm{~g}$ de extrato de levedura e $100 \mu \mathrm{L}$ Tween 80 (Dallastra, 2016). A concentração dos conídios foi determinada em câmara de Neubauer, padronizada em $10^{7}$ conídios/mL e reservada para posterior inoculação dos substratos.

Preparo dos Meios de Cultivo: $\mathrm{O}$ arroz tipo I foi cozido por imersão em água à $80^{\circ} \mathrm{C}$ durante 4 minutos, e posteriormente, deixado à temperatura ambiente por duas horas sobre papel kraft até atingir de $40 \%$. O bagaço do coco de babaçu foi deixado descongelar até a temperatura ambiente. 
Fermentações: Em erlenmeyers tampados foram colocados $10 \mathrm{~g}$ de substrato e os conjuntos (erlenmeyer+substrato) foram esterilizados em autoclave à temperatura de $120{ }^{\circ} \mathrm{C}$ por 20 minutos. Posteriormente, o conjunto foi deixado resfriar até a temperatura ambiente e foi inoculado $1 \mathrm{~mL}$ da solução nutriente e da suspensão inoculante. $\mathrm{O}$ sistema foi mantido em incubadora $\mathrm{BOD}$ a $28^{\circ} \mathrm{C}$ por 14 dias.

Extração da enzima: Uma vez concluída a fermentação, a enzima foi extraída do material fermentado com uma solução composta por $20 \mathrm{~mL}$ de tampão McIlvaine $\mathrm{pH}$ 7,0 e $0,25 \%$ de espelhante adesivo Tween 80 . A mistura de sólido fermentado (10 g) e a solução extratora $(20 \mathrm{~mL})$ foram submetidas à agitação manual até homogeneização e desprendimento dos conídios que continham as lipases na sua superfície. Em seguida, o material foi filtrado em TNT, conforme a Figura 1. Esse filtrado ou extrato enzimático bruto foi congelado até a determinação de atividade lipolítica.

Figura 1- Extrato bruto enzimático sendo filtrado.

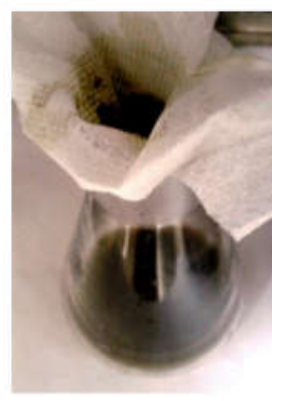

Atividade enzimática: A atividade lipolítica foi determinada de acordo com o método descrito por Freire et al. (1997) com algumas alterações. Em frascos erlenmeyer de $125 \mathrm{~mL}$ foram adicionados $19 \mathrm{~mL}$ de emulsão (1\% de triton X-100 e 5\% de azeite de oliva) em tampão McIlvaine pH 7,0. Essa emulsão foi homogeneizada em shaker por 3 min a $37^{\circ} \mathrm{C}$ e $200 \mathrm{rpm}$, em seguida foi adicionado $1 \mathrm{~mL}$ do extrato enzimático bruto e incubada por $30 \mathrm{~min}$ a $28^{\circ} \mathrm{C}$ e $200 \mathrm{rpm}$. Após a incubação, a reação foi detida com $20 \mathrm{~mL}$ de solução de acetona: etanol 1:1 (v/v) e os ácidos graxos liberados foram titulados com solução de $\mathrm{NaOH} 0,1 \mathrm{M}$ até pH final 11. A atividade enzimática (U. mL-1) foi determinada de acordo com a Equação 1:

Atividade.enzimatica $=\frac{\left(V t_{f}-V t_{i}\right) \times 100 \times F d \times M}{t}$

Onde:

$V t_{f}$ é o volume de $\mathrm{NaOH}$ após a reação, $V t_{i}$ é o volume de $\mathrm{NaOH}$ usado para titular o branco, $M$ é concentração normal de $\mathrm{NaOH}, t$ é o tempo total de reação e $F d$ é o fator de diluição.

Uma unidade de atividade lipolítica foi definida como a quantidade de enzima que libera $1 \mu \mathrm{mol}$ de ácido graxo por minuto, nas condições descritas.

Planejamento experimental e análise estatística: Os experimentos foram realizados em delineamento inteiramente casualizado, sendo as médias dos tratamentos comparadas pelo 
teste de Tukey, ao nível de 5\% de significância. As análises estatísticas foram realizadas utilizando o programa Computacional Assistat, versão 7.7. Os níveis das variáveis analisadas se encontram na Tabela 1 . No total foram realizados 06 tratamentos, com 03 repetições, para um total de 3 parcelas.

Tabela 1. Concentrações dos substratos.

\begin{tabular}{ccc}
\hline Tratamento & $\begin{array}{c}\text { Arroz } \\
\text { tipo I (g) }\end{array}$ & $\begin{array}{c}\text { Bagaço do } \\
\text { coco babaçu } \\
\text { (g) }\end{array}$ \\
\hline A & 10 & 0 \\
\hline B & 8 & 2 \\
\hline C & 6 & 4 \\
\hline D & 4 & 6 \\
\hline E & 2 & 8 \\
\hline F & 0 & 10 \\
\hline
\end{tabular}

\section{RESULTADOS E DISCUSSÃO}

Os resultados apresentados na Figura 2 mostraram que a maior atividade enzimática obtida foi para o tratamento "A", aquele realizado unicamente com arroz tipo I, sendo esta de 8,33 U. mL-1. Este valor é superior ao obtido por Silva et al. (2005), no qual a atividade lipolítica foi de 4,48 U. mL-1 para um sistema de fermentação submersa utilizando o arroz como substrato.

Figura 2 - Media da atividade enzimática e desvio padrão.

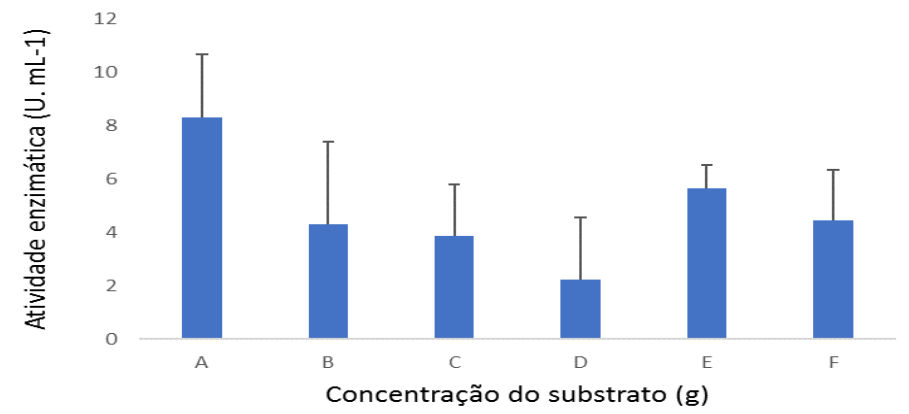

No tratamento "E" ( $80 \%$ do bagaço do babaçu mais $20 \%$ de arroz tipo I) também houve uma atividade enzimática considerável, de 5,57 U. mL-1, onde o fungo $M$. anisopliae apresentou uma boa adaptação ao bagaço do coco babaçu. Cordova et al., (1998) obteve 1,73 U. mL-1 utilizando bagaço de cana e o agente fermentador Rhizomucor pusillus, já Fleuri et al. (2014) obteve uma atividade enzimática de 14.4 U.mL-1 através do fungo Aspergillus sp. utilizando resíduo agroindustrial (farelo de trigo) ambos os autores utilizaram o processo de FES, confirmando assim o potencial que os resíduos agroindustrais possuem como meios de cultura para a produção de enzimas de alto valor agregado.

O valor de atividade lipolítica para o tratamento "F", foi de 4,44 U.mL-1, quando utilizado somente o resíduo do coco do babaçu. Pinheiro (2006) realizou um experimento em fermentação submersa e obteve um valor de atividade lipolítica de 3,15 U.mL-1 em meio sintético, além de ser uma atividade menor, o custo de produção se eleva devido ao alto custo 
do meio, o que reduziria a possibilidade de produzir a enzima em escala industrial através deste processo. Ao se comparar a produção de lipase por resíduos em meio sintético observase que, o meio de fermentação composto por resíduos agroindustrais teve maior atividade lipolítica, demostrando a viabilidade da produção de lipase. O resíduo agroindustrial como substrato oferece suporte nutricional e de crescimento ao microrganismo durante a fermentação, a FES apresenta-se como um processo mais vantajoso e de baixo custo em relação à fermentação submersa, além de ter altos rendimentos e proporciona uma facilidade na recuperação dos produtos gerados (Sharma et al., 2001).

Em termos estatísticos, a ANOVA revelou efeito não significativo para os tratamentos (Tabela 2), indicando que não existem diferenças significativas entre os mesmos. Este fato sugere que já que não existe diferença estatística entre os tratamentos, o bagaço do coco babaçu é um substrato promissório para a produção de lipases, devido ao baixo custo e às vantagens ambientais, onde o resíduo é utilizado em vez de ser descartado no meio ambiente.

Tabela 2. ANOVA para a atividade lipolítica.

\begin{tabular}{|l|l|l|l|l|}
\hline FV & GL & QM & F & P \\
\hline Tratamentos & 5 & 10,0104100 & 2,0557 & $0,1483^{*}$ \\
\hline Resíduo & 11 & 4,8695 & & \\
\hline Total & 16 & 10,3616580 & & \\
\hline CV\% & 47,88 & & & \\
\hline
\end{tabular}

* Não significativo ao nível de $5 \%$ de probabilidade $(\mathrm{p}>=0,05)$

Os resultados demonstraram que foi possível produzir lipases pelo processo de fermentação em estado sólido utilizando o bagaço do coco de babaçu, porém será necessário realizar estudos mais aprofundados, os quais incluam maior variação nas concentrações de substrato e produção em uma escala maior, primeiramente em escala piloto e depois em larga escala, vencendo os desafios próprios da ampliação de escala.

\section{CONCLUSÃO}

Foi possível produzir as lipases por fermentação em estado sólido através do fungo $M$. anisopliae utilizando arroz tipo I e bagaço do coco babaçu como substratos. Os resultados desses experimentos poderiam viabilizar a produção de lipases com baixo custo, porém será necessário realizar estudos mais aprofundados, os quais incluam maior variação nas concentrações de substrato e produção em uma escala maior, primeiramente em escala piloto e depois em larga escala, vencendo os desafios próprios da ampliação de escala.

\section{REFERÊNCIAS}

CARRAZZA, L. R.; SILVA, M. L.; ÁVILA, J. C. C. Manual Tecnológico de Aproveitamento Integral do Fruto do Babaçu. Brasília - DF. Instituto Sociedade, População e Natureza (ISPN). Brasil, 2012.

CASTILHO, L. R.; POLATO, C. M. S.; BARUQUE, E. A.; SANTANNA JR., G. L.;FREIRE, D.M.G. Economic analysis of lipase production by Penicillium restrictum in solid state and submerged fermentations. Biochemical Engineering Journal, v. a, p. 239- 
247, 2000.

COLLA, L. M.; REINEHR, C. O.; COSTA, A. V. Aplicações e produção de lipases microbianas., Revista CIATEC - UPF, v. 4, p.1-14, 2012.

CORDOVA, J.; NEMMAOUI, M.; ISMAILI-ALAOUI, M.; MORIN, A.; ROUSSOS, S.; RAIMBAULT, M.; BENJILALI B. Lipase production by solid state fermentation of olive cake and sugar cane bagasse. Journal of Molecular Catalysis B: Enzymatic v.5, p.75-78, 1998.

DAllaStRA, E. D. G.; Produção de Metarhizium anisopliae utilizando arroz tipo i, quirela de arroz, farelo de soja e farelo de trigo como substrato. Dissertação (Curso de Engenharia de Alimentos), Universidade Federal do Tocantins, Palmas, 2016.

FLEURI LF.; de Oliveira MC.; Arcuri MLC.; Capoville BL.; Pereira MS. Production of Fungal Lipases Using Wheat Bran and Soybean Bran and Incorporation of Sugarcane Bagasse as a Co-substrate in Solid-state Fermentation. Food Sci Biotechnol 23: 11991205, 2014.

FREIRE, D. M. G.; GOMES, M. P.; BOM, S. P. E. Lipase production by a new promising strain of Penicillium restrictum; Journal of the Brazilian Society for Microbiology, v. 28, p. 6-12, 1997.

MAHADIK, N. D.; PUNTAMBEKAR, U. S.; BASTAWDE, K. B.; KHIRE, J. M.; GOKHALE, D.V. Production of acidic lipase by Aspergillus niger in solid state fermentation. Process biochemistry, v. 38, p. 715-721, 2002.

OLIVEIRA M. M.;L. M. PINOTTI. Produção de lipases por rhizomucor sp. Departamento de Engenharias e Tecnologia, Universidade Federal do Espírito Santo, COBEC, 2015.

PINHEIRO, T. L. F. Produção de lipases por fermentação em estado sólido e fermentação submersa utilizando Penicillium verrucosum como microrganismo,Dissertação (Departamento de Ciências Agrárias) Universidade Regional Integrada, Erechim, Rio Grande do Sul, 2006.

SANTANA, R. S. M. Produção de enzimas amiloliticas através da fermentação em estado sólido. 2012. 73f. Dissertação (Pós-Graduação em Engenharia de Alimentos) Universidade Estadual do sudoeste da Bahia, Bahia, 2012.

SAXENA, R.K.; GHOSH, P.K.; GUPTA, R.; DAVIDSON, W.S.; BRADOO, S.; GULATI, R. Microbial lipases: potential biocatalysts for the future industry. Curr. Sci. 77:101-115, 1999.

SHARMA, R.; CHISTI, Y.; BANERJEE, U.C. Production, purification, characterization, and applications of lipases. Biotechnology Adv., v. 19, p. 627-662, 2001.

SHAUKAT A.; ZHEN H.; REN X. S.; BASHIR M. H.; AFZAL M.; TONG L. Production and Extraction of Extracellular Lipase from Entomopathogenic Fungus Metarhizium anisopliae (Clavicipitaceae: Hypocreales). Pakistan J. Zool., v. 41(5), pp. 341-347, 2009.

SILVA, W.O.B.; MITIDIERI, S.;SCHRANK A.; VAINSTEIN M. H. Production and extraction of an extracellular lipase from the entomopathogenic fungus Metarhizium anisopliae. Process Biochemistry v.40, p.321-326, 2005.

SILVA, W.O.B., Lipases de Metarhizium anisopliae: purificação parcial, regulação e secreção durante o processo de infecção do carrapato bovino Boophilus microplus; Dissertação(Programa de pós-graduação em biologia celular e molecular), Universidade Federal do Rio Grande do Sul, Porto Alegre, 2005. 